\title{
Minimizing Stimulus Current in a Wearable Pudendal Nerve Stimulator Using Computational Models
}

\author{
Arsam N. Shiraz, Student Member, IEEE, \\ Michael Craggs, Brian Leaker, and Andreas Demosthenous, Senior Member, IEEE
}

\begin{abstract}
After spinal cord injury, functions of the lower urinary tract may be disrupted. A wearable device with surface electrodes which can effectively control the bladder functions would be highly beneficial to the patients. A trans-rectal pudendal nerve stimulator may provide such a solution. However, the major limiting factor in such a stimulator is the high level of current it requires to recruit the nerve fibers. Also, the variability of the trajectory of the nerve in different individuals should be considered. Using computational models and an approximate trajectory of the nerve derived from an MRI study, it is demonstrated in this paper that it may be possible to considerably reduce the required current levels for trans-rectal stimulation of the pudendal nerve compared to the values previously reported in the literature. This was corroborated by considering an ensemble of possible and probable variations of the trajectory. The outcome of this study suggests that trans-rectal stimulation of the pudendal nerve is a plausible long term solution for treating lower urinary tract dysfunctions after spinal cord injury.
\end{abstract}

Index Terms-Electrode configuration optimization, finite element model (FEM), hybrid computational model, pudendal nerve stimulation.

\section{INTRODUCTION}

$\mathbf{T}$ HE primary functions of the lower urinary tract (LUT) are urine storage and voiding. These functions depend on the coordinated activities of the bladder muscle (detrusor) and an outlet consisting of the bladder neck and urethral musculature. This coordination is mediated by the neural signals conveyed to and from the central nervous system and the periphery of the body through peripheral nerves. Autonomic, via pelvic and hypogastric nerves, and somatic, via pudendal nerve, peripheral nerves are involved in the innervation of the LUT through their afferent and efferent pathways [1].

After spinal cord injury (SCI), depending on the level, duration and completeness of the lesion, the normal functions of

Manuscript received June 13, 2015; revised August 30, 2015; accepted August 30, 2015. Date of publication September 22, 2015; date of current version April 06, 2016. This work was supported by a Ph.D. scholarship to A. N. Shiraz from University College London and Nephro-Urology Clinical Trials (NUCT) Ltd. Corresponding author: Andreas Demosthenous (e-mail: a.demosthenous@ucl.ac.uk).

A. N. Shiraz and A. Demosthenous are with the Department of Electronic and Electrical Engineering, University College London, London WC1E 7JE, U.K. (e-mail: a.shiraz@ucl.ac.uk; a.demosthenous@ucl.ac.uk).

M. Craggs is with the University College London Medical School, London WC1E 6BT, U.K. (e-mail: michael.craggs@ucl.ac.uk).

B. Leaker is with Queen Anne Street Medical Centre, London W1G 8HU,

U.K. (e-mail: brian.leaker@heartlungcentre.com).

Color versions of one or more of the figures in this paper are available online at http://ieeexplore.ieee.org.

Digital Object Identifier 10.1109/TNSRE.2015.2480755 the LUT may be disrupted. Neurogenic detrusor overactivity (NDO), causing incontinence, and detrusor sphincter dyssynergia (DSD), leading to inefficient voiding, are the two common conditions arising after SCI [2]. Inadequate management of the LUT dysfunctions after SCI may have fatal consequences. Prevalent treatment and management of these conditions uses a combination of drugs to suppress NDO and intermittent catheterization for voiding. However, the troublesome side effects of the drugs and frequent episodes of LUT infection due to catheterization demonstrate the need for alternative forms of treatment and management [3]. The alternative should be efficacious and address patient concerns [4]. This combination will ensure that the quality of life is increased for patients.

Applying stimulus current to the central and peripheral neurons to use the intact segments of the neural circuits may provide such an alternative. The Finetech-Brindley (Finetech Medical Ltd., Welwyn Garden City, U.K.) device for sacral anterior root stimulation is one example used to achieve clinically efficient voiding [5]. This solution requires dorsal rhizotomy to be effective which has irreversible consequences. Another example of such devices is InterStim (Medtronic plc, Dublin, Ireland) for sacral root stimulation used to improve continence. The surgical operations associated with these solutions are costly and expose patients to the associated risks.

Given the converging pathways of the pudendal nerve and autonomic vesical nerves [6], pudendal nerve stimulation in various positions along its course has been a topic of research for controlling the LUT function after SCI. Various studies [7]-[9] have demonstrated the possibility of modulating vesical behavior depending on the position and frequency of the stimulation along the course of the pudendal nerve and its branches. This effect has essentially been attributed to the modulatory effect of the pudendal afferent input on the autonomic vesical nerves [1]. Craggs et al. [10], [11] in a study on six SCI patients suffering from NDO and DSD demonstrated the feasibility of conditional trans-rectal pudendal nerve neuromodulation to inhibit NDO. The electromyographic (EMG) signal from the external anal sphincter was used as the control signal for stimulation. Such a wearable device can potentially improve the quality of life for the patients. However, to induce the inhibition of NDO, high levels of applied stimulus current were reported in some cases. This may render the solution impractical as high levels of stimulus current increases the chances of tissue and electrode damage and other structures and nerves in the vicinity may be stimulated which may result in unforeseen responses.

Other studies based on computational models of intraurethral electrical stimulation of the pudendal nerve, with different elec- 
trode sizes and shapes [9] and using a cuff multi-electrode on the proximal pudendal nerve trunk [8], have investigated the possibility of achieving selectivity and reducing stimulus threshold. However, no such study has investigated the dynamics of neural activation for trans-rectal stimulation of the pudendal nerve as presented here.

In this paper, based on a magnetic resonance imaging (MRI) study and the existing literature, a finite element model (FEM) of the pelvic region and trans-rectal stimulator was developed. The model was used in tandem with a model of the mammalian axon to investigate the effect of various electrode configurations on the required stimulus current along the main nerve trunk. However, in cases where the nerve is not accessed directly, relative variations of the course of the nerve with respect to the stimulation electrodes should be taken into account. These variations might be anatomical across patients or due to the movements of the structures in the vicinity. Using an ensemble of randomly generated, yet anatomically valid nerve courses, the possibility that the stimulus current could be minimized in each case by means of an array of electrodes on the trans-rectal stimulator, was examined. It was demonstrated that for an ensemble of ten possible nerve courses and the course based on the MRI study, a particular electrode configuration requires considerably lower stimulus current to excite the target nerve. This result suggests that a specific configuration can be used for different patients and for a patient in different postures while relatively low current levels are required.

The rest of the paper is organized as follows. In Section II, the methods in the MRI study, making the finite element model and the axon model are presented. In Section III, the results based on the computational models are shown. After discussion of the results in Section IV, concluding remarks are presented in Section V.

\section{METHODS}

\section{A. MRI Study}

To find an approximate course of the pudendal nerve and identify the main anatomical features and their relative distances with respect to the stimulator in situ, an MRI study was performed. Originating from either side of the sacral cord, the pudendal nerve enters the gluteal region through the lower part of the sciatic foramen. Turning around the sacrospinous ligament near its attachment to the ischial spine, the pudendal nerve re-enters the perineum via the lesser sciatic foramen. Running along the distal edge of the ischioanal fossa, the nerve passes in the vicinity of the ano-rectal junction [12]. The MRI study intended to identify an approximate course of the nerve in this last section.

With the approval of the local research and development review committee, a trans-rectal stimulator as shown in Fig. 1 was used for an MRI study on a male volunteer. The stimulator was similar to the one used by Craggs et al. [10]. It was made from silicone rubber and the electrodes were medical grade stainless steel disks. The metallic electrodes were removed and water based gel was injected in the position of the cathodes to make their positions visible in the MRI scans. Using a $1.5 \mathrm{~T}$ MRI instrument, a set of transverse and sagittal MRI scans was taken

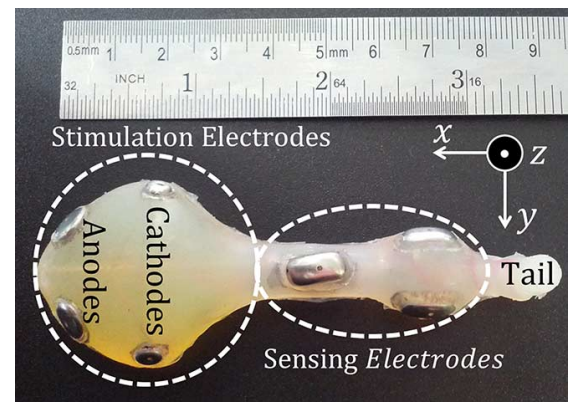

Fig. 1. Trans-rectal stimulator used in the MRI study. This stimulator was also used by Craggs et al. [10] using the electrodes based on the functionalities annotated in the picture.

of the subject with the stimulator in situ as shown in Fig. 2. Syngo fastView software (Siemens Healthcare, U.K.) was used to study the MRI scans. The approximate positions of the cross sections of the pudendal nerve at the edge of the ischoanal fossa were marked in the transverse planes as shown in Fig. 2(a) in nine slides (only three shown here). Different anatomical features and their respective size and position were noted based on transverse and sagittal planes.

All the measurements were taken by defining a Cartesian coordinate system whose origin was placed inside the probe between the two cathodes. The axes were defined consistent with those shown in Fig. 1. The coordinates of the mentioned cross sections were then derived. By cubic spline interpolation of the cross sections found in the MRI study, a smooth and densely sampled course of the nerve was then defined in MATLAB R2014a (MathWorks, Inc., Natick M, USA). The pudendal nerve is paired as it symmetrically runs on the left and right side of the pelvis. The study presented in this paper only considers the course of the nerve on the right side of the body due to symmetry.

\section{B. Volume Conductor Model}

Based on the MRI study, an FEM of the stimulator and the pelvic region was constructed as shown in Fig. 3 in COMSOL Multiphysics 4.3 (COMSOL, Ltd., Cambridge, U.K.). Apart from the main anatomical features around the stimulator in situ, three main tissue layers around it, each of which composed of structures of relatively similar conductivities, were considered. Layer 1 , immediately around the stimulator, is the intestinal tissue and the levator ani muscle. Layer 2 represents the fatty tissue of the ischioanal fossa and connective tissue between organs. Layer 3 is the pelvic floor musculature. All the subsequent simulations were run on a computer with $3.4 \mathrm{GHz}$ Intel i7-2600 CPU and 16 GB RAM.

A quasi-static approximation of Maxwell's equations as shown in (1) was used to simulate the electric potential in the model. This was implemented in COMSOL as discussed in Appendix A.

$$
\nabla \cdot \sigma \nabla V=0
$$

where $\sigma$ is the conductivity of the medium and $V$ is the extracellular potential in the model. 

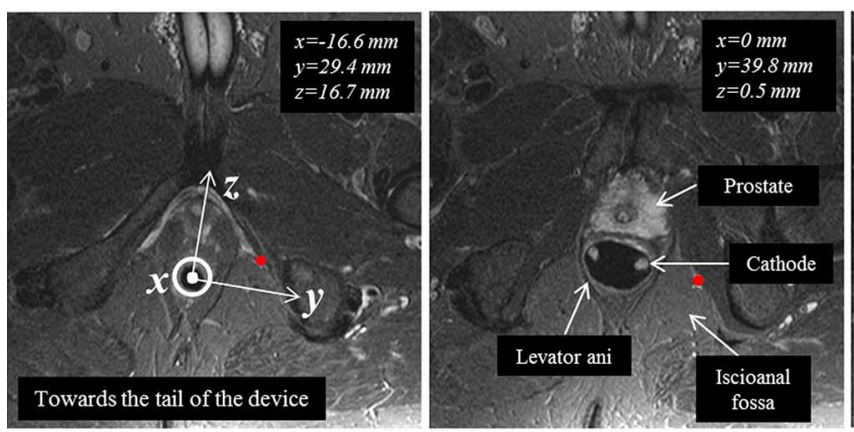

(a)

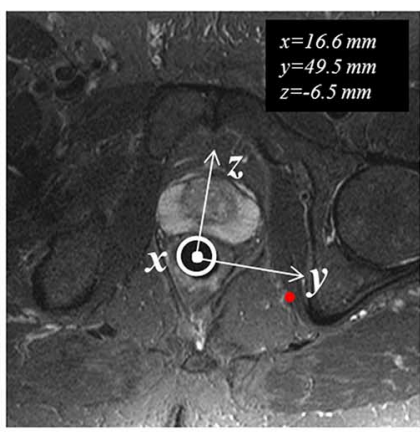

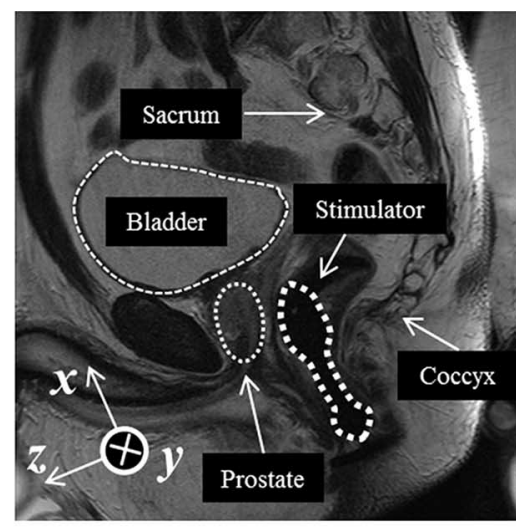

(b)

Fig. 2. (a) Transverse MRI slides of the pelvic region with the trans-rectal stimulator in situ, showing the cross section of the pudendal nerve at the edge of the ischoanal fossa with red circles. The coordinates of each cross section, cathodes, and different anatomical features are shown. (b) Sagittal MRI slide of the pelvic region with the stimulator in situ, showing different anatomical features in the vicinity.

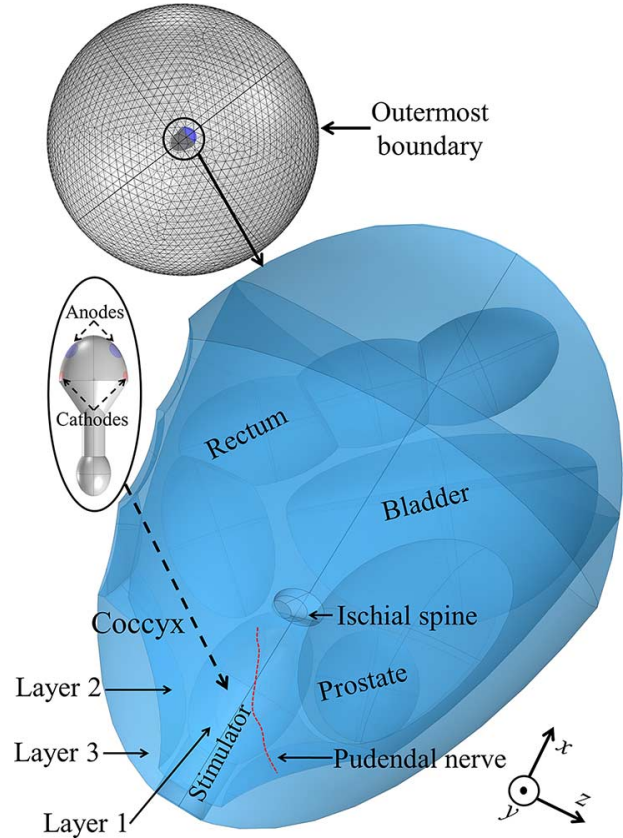

Fig. 3. FEM of the trans-rectal stimulator in Fig. 1 in situ. Different anatomical features in the model are shown. An electrode configuration similar to the one in [10] was used.

1) Boundary Conditions and Discretization: A sphere was defined around the model as shown in Fig. 3 and Dirichlet boundary condition $(V=0)$ was applied to the external boundaries of the mentioned sphere. This would implement an approximation of ground at infinity. Given the insulation at the boundary of the body, this medium was set to be nonconductive $\left(\sigma=10^{-10} \mathrm{~S} / \mathrm{m}\right)$. Since the current spread is mainly localized in layer 1 and spreads slightly in layer 2 , as shown in Section II-B2, ignoring the other body structures is assumed not to affect the modelling outcome. For implantable devices, the conductivity of the engulfing medium is generally set to that of saline [13]. However, given the size of the stimulator and that the external boundary of the model extends almost to the boundaries of the body, this medium was set as insulation. The model was discretized using nonuniform free tetrahedral elements, while it was more finely meshed in layers 1 and 2 by adjusting the maximum element size. This resulted in a total of about 1.2 M elements (about 1.6 M degrees of freedom).

The electrodes were defined as equipotential surfaces on which the current density distribution was nonuniform. In all the following cases, anodes were set as the source of a total of $1 \mathrm{~mA}$ while the same amount was set to sink in cathodes. This was implemented in COMSOL by Terminal current of negative value for the cathodes and positive value for the anodes. The electrode-tissue interface contact impedance was ignored based on the experimental measurements [14]. Appropriate continuity conditions were implemented at the boundaries of different media [15].

After adjusting the conductivity of different media as shown in Section II-B2, the radius of the external sphere was changed from $50 \mathrm{~cm}$ to $200 \mathrm{~cm}$ in steps and the resulting simulated electric potential along the trajectory of the nerve was recorded. These variations would only introduce a shift in the electrical potential along the nerve. When the radius of the external sphere was set to $90 \mathrm{~cm}$, even doubling the radius size would result in less than $1 \%$ change in the result. Thus, the radius of the external sphere was set to $90 \mathrm{~cm}$.

By adjusting the maximum element size in layers 1 and 2, the number of elements was changed from about $1.2 \mathrm{M}$ to 7 $\mathrm{M}$ in steps. The results, in terms of the electric potential along the nerve, changed only by about $1 \%$ while the simulation time at the lowest discretization level was 3\% of that of the highest. Above this discretization level, the computer ran out of memory. Thus, approximately $1.2 \mathrm{M}$ element count size was considered to be sufficient.

2) Conductivities: The body of the stimulator was set to be nonconductive $\left(\sigma=10^{-10} \mathrm{~S} / \mathrm{m}\right)$. A range of values is reported for the conductivity of muscle and fat at low frequencies. The conductivity of layer 1 in the model was set between $0.2 \mathrm{~S} / \mathrm{m}$ and $0.4 \mathrm{~S} / \mathrm{m}$ in steps, and the conductivities of the rest of the components of the model were set to their typical values. The corresponding simulated maximum voltage drop between the electrodes was recorded in each case. Similarly, the conductivity of layer 2 was set to $0.03 \mathrm{~S} / \mathrm{m}$ and $0.05 \mathrm{~S} / \mathrm{m}$ and the maximum simulated voltage drop was recorded. The variation in the maximum 
TABLE I

CONDUCTIVITIES USEd IN VOLUME CONDUCTOR MODEL

\begin{tabular}{ccc}
\hline \hline Model component & Structure & $\begin{array}{c}\text { Reported conductivity S/m } \\
\text { (selected conductivity) }\end{array}$ \\
\hline $\begin{array}{c}\text { Layer 1 (Intestinal } \\
\text { tissue, muscle) }\end{array}$ & $\begin{array}{c}\text { Rectal/anal wall and } \\
\text { levator ani muscle }\end{array}$ & $\begin{array}{c}0.2-0.4[16]-[19] \\
(0.4)\end{array}$ \\
\hline $\begin{array}{c}\text { Layer 2 } \\
\text { (connective } \\
\text { tissue, fat) }\end{array}$ & Ischioanal fossa & $\begin{array}{c}0.03-0.05[16]-[19] \\
(0.04)\end{array}$ \\
\hline Layer 3 (muscle) & $\begin{array}{c}\text { Pelvic floor } \\
\text { musculature } \\
\text { (obturator muscle) }\end{array}$ & $\begin{array}{c}0.2-0.4[16]-[19] \\
(0.4)\end{array}$ \\
\hline Prostate & Prostate & $\begin{array}{c}0.4[16]-[19] \\
(0.4)\end{array}$ \\
\hline Bladder & Bladder & $\begin{array}{c}0.2[16]-[19] \\
(0.2)\end{array}$ \\
\hline Rectal content & Feces or gas & $\begin{array}{c}0.1 / 10^{-5}[20] \\
\left(0.1 / 10^{-5}\right)\end{array}$ \\
\hline
\end{tabular}

voltage drop due to variations in the conductivity of layer 1 was $214.5 \mathrm{~V} / \mathrm{S}$ while variations in the conductivity of layer 2 resulted in $15.78 \mathrm{~V} / \mathrm{S}$ of variation in maximum voltage drop. This confirms that current is mainly confined to layer 1 . The conductivity of layer 2 and other components in the model were set to their typical values and the conductivity of layer 1 was swept across a range reported in the literature. A conductivity resulting in the maximum voltage drop across electrodes consistent with previous experimental measurements [14] was assigned to layer 1. All the conductivities were assumed to be isotropic. Table I summarizes the conductivities of different components of the model.

The steps in Sections II-B1 and II-B2 were performed iteratively to arrive at the final conductivity values [16]-[20].

\section{Nerve Model}

Myelinated afferent fibers in the pudendal nerve were modeled as double layer cable models with explicit representation of nodes of Ranvier with active and passive properties and compartments with only passive properties as in [21]. The compartments with passive properties between every two active nodes included two myelin attachment segments, two paranodal segments, and ten internodal segments.

The fiber diameters based on the contribution by Schalow et al. [22] were set to follow a normal distribution of $12 \mu$ m mean with a standard deviation of $1 \mu \mathrm{m}$. The geometric properties presented in [21] were linearly interpolated proportional to the fiber diameter to derive the values.

In Matlab, a fiber diameter value within the distribution stated above was generated. A node of Ranvier was then randomly placed between 0 and $\Delta x$ of the arc-length of the course of the nerve found in the MRI study, where $\Delta x$ was the corresponding node to node distance for the mentioned fiber diameter. All the passive compartments, whose geometric parameters were found by linear interpolation based on the fiber diameter, were placed along the arc-length between every subsequent node in sequence until no more compartments could be added. The fiber was terminated by a node. These steps were repeated 100 times for this approximately 5 -cm-long nerve section.

The geometric properties of all the fibers and the number of compartments and their position along the arc-length of the nerve were exported to Neuron 7.3 [23] to create a 100 double layer cable model of myelinated axon with imperfect insulation of the myelin sheath. All the electrical parameters of the model were used as in [21]. The underlying differential equations, defining active properties of the channels in the nodes based on the work in [21] were extracted from the files on Model DB [24] with accession number 3810. Backward Euler integration with a time step of $10 \mu \mathrm{s}$ was used to run the simulations. For all the compartments in the models, the number of segments was set to one (i.e., $n s e g=1$ ) and unless otherwise stated the simulation was run for $10 \mathrm{~ms}$.

Although the model used for axons and the corresponding parameters were originally intended to simulate motor fibers, there is evidence in the literature that it simulates afferent fibers [13]. Early research demonstrates that for stimulus pulses of $200 \mu \mathrm{s}$ duration efferent and afferent fibers have similar responses [25].

\section{Hybrid Model}

Coupling the FEM results with the Neuron model, it was possible to simulate the response of the nerve to extracellular potential generated by the trans-rectal stimulator. The electric potential vector along the nerve was exported to Matlab from COMSOL. This vector was interpolated and assigned to every compartment. A time vector and a potential vector were constructed to generate $200 \mu$ s stimulus pulses as used in [10]. To simulate various stimulus current levels, the potential vector was multiplied. This is valid under quasi-static approximation [13].

The time and potential vectors were exported to the Neuron model and applied as extracellular potential in each compartment (i.e., e_extracellular). For various applied current steps, not larger than $10 \mathrm{~mA}$, the percentage of the fibers activated (PA) ( $n=100)$ along the trajectory of the nerve derived in the MRI study, was recorded between 0 and $100 \%$. Activation was defined as observing action potential in the first and last node of a fiber.

In the model, variations of the position of the fascicle containing the fibers in the nerve and variations of the position of the fibers within a fascicle were ignored as a more extensive set of variations is explored in this paper. Also, since the epineurium (EP) engulfing the fascicles is composed of a fatty tissue, and given that the nerve is passing through the fatty tissue of the ischioanal fossa in the area under study, the distinction between the two fatty tissues was not made. Furthermore, endoneurium (EN) and perineurium (PE) tissue layers in and around the fascicles were ignored. EN has distinct anisotropic conductivity (longitudinal $=0.571 \mathrm{~S} / \mathrm{m}$, transverse $=0.083 \mathrm{~S} / \mathrm{m}$ ). PE, surrounding each fascicle and constituting $3 \%$ of the fascicle diameter, has a conductivity of only $0.002 \mathrm{~S} / \mathrm{m}$ [26]. The dimensional ratios and the fact that the nerve is not placed in a single plane would increase modelling complexity and the computation time would increase considerably if these features were to be incorporated.

To verify to what extent these assumptions change the results, a line, connecting the first and last cross sections found in the MRI study, was formed to approximate the nerve. A cylinder of $4 \mathrm{~mm}$ diameter was defined around the linear approximation of the nerve to represent EP. Another cylinder of $300 \mu \mathrm{m}$ diameter was defined around the nerve to represent EN [8]. A thin layer of $9 \mu \mathrm{m}$ was defined around EN to represent PE. This was done in COMSOL using Contact Impedance. Then, four more fascicles 


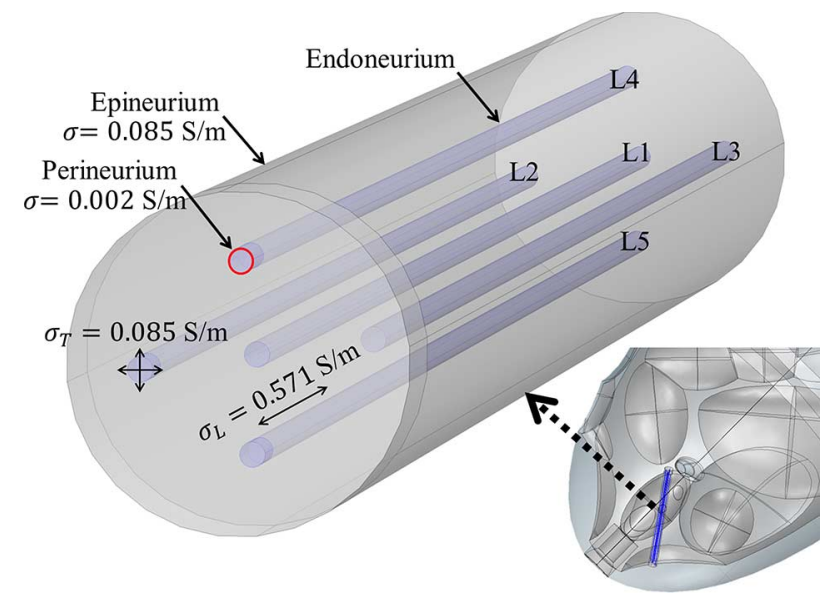

Fig. 4. Linear approximation of the nerve and different tissue layers for five fascicles. The most central fascicle is the one that the trajectory derived based on the MRI study lies within.

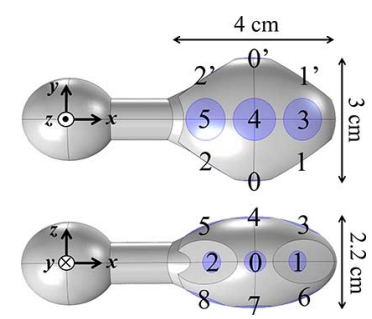

Fig. 5. Modified design of the stimulator to accommodate more electrodes. The naming convention used throughout the paper is shown.

on four corners of the EP were defined as shown in Fig. 4. To set the conductivity of EN, a conductivity tensor was defined by defining a local coordinate and one of its axes was defined in the direction of the nerve as shown in Appendix B.

For various applied current levels in $10 \mathrm{~mA}$ steps, the PA ( $n=100$ ) along the linear approximation of the nerve without $\mathrm{EP}, \mathrm{PE}$, and EN was recorded. Then, the percentage of the fibers activated along the linear approximations of the nerve at the center and four corners of EP with PE, and EN in place were simulated.

\section{E. Optimization}

As shown in Fig. 5, the stimulator was modified to accommodate more electrodes on it, compared to the stimulator in Fig. 1. The primed electrodes (i.e., $0^{\prime}, 1^{\prime}$, and $2^{\prime}$ ) were facing the left side of the body and were not included in the subsequent study. This is due to the symmetry of the lateral branches of the pudendal nerve as mentioned in Section II-A. The diameters of electrodes 0 to 2 were $0.5 \mathrm{~cm}$ and those of electrodes 3 to 8 were $1 \mathrm{~cm}$ as they were shared between the lateral sides of the stimulator. In all the investigations presented in this paper, the naming convention follows a cathode-anode format (e.g., 01 means setting electrode 0 as cathode and electrode 1 as anode). Electrode 0 was placed in a position facing the nerve and was used as cathode or anode in all the selected configurations leading to a total of 16 possible configurations to generate the field in the vicinity of the nerve. The configurations in which electrode 0 is used as cathode are categorized as forward configurations.
Those configurations in which electrode 0 is used as anode are referred to as reversed configurations in the paper.

1) Activating Function $(A F)$ : To have a heuristic view of the possible positions of depolarization and hyperpolarization along the course of the nerve, the normalized activating function (AF) [27] defined as the second spatial difference of extracellular electric potential along the course of the nerve as shown in (2) was calculated. AF was formed in Matlab for 100 axons randomly placed along the trajectory of the nerve with normal distribution of fiber diameter, as mentioned before, for all the possible bipolar electrode configurations. For reversed configurations, to find the extracellular electric potential along the nerve, the electric potential simulated in FEM was multiplied by -1 to reduce the number of simulation runs.

$$
\mathrm{AF}=\frac{\Delta^{2} V_{e}}{\Delta l^{2}}=\frac{V_{e}(n-1)-2 V_{e}(n)+V_{e}(n+1)}{\Delta x^{2}}
$$

where $l$ is the arc-length of the nerve, $V_{e}(n)$ is the extracellular potential on the $n$th node of the myelinated fiber, and $\Delta x$ is the node to node distance. For the first and last nodes the first spatial derivative was used [9].

2) Dynamic Axon Response: For all 16 possible configurations, the $\mathrm{PA}$ versus the required current was recorded for equally spaced current steps. The maximum current step was $10 \mathrm{~mA}$.

For the configuration requiring the lowest current level, the PA versus current was investigated for ten 100-axon random populations to identify the effect of the population on the results. For the same configuration, the PA for the current level leading to $100 \%$ activation for the initial pulse was simulated for ten consecutive pulses, repeated at $35 \mathrm{~Hz}$ and $5 \mathrm{~Hz}$. These are the levels reported in the literature for which different neuromodulation effects are observed [9].

\section{F. Nerve Variations}

The course and branching of the pudendal nerve has been shown to be variable in different individuals [28], [29]. Also, the trajectory of the nerve with respect to the trans-rectal stimulator may be changing in different body postures. Thus, any optimization study should consider these variations. The variation of the re-entrance position of the nerve into the perineum has been quantified with respect to ischial spine in cadaveric and ultrasound studies [28], [29]. Four points as shown in Fig. 6 were selected along the trajectory of the nerve found in the MRI study. A variation pattern as defined in (3) was implemented (e.g., $a_{x} \pm 1$ means that the $x$ component of point $a$ can be changed uniformly randomly between -1 and +1 )

$$
\left[\begin{array}{ccc}
a_{x} \pm 1.5 & a_{y}-1 & a_{z} \pm 1.5 \\
b_{x} \pm 1 & b_{y}-1 & b_{z} \pm 1 \\
c_{x} \pm 1 & c_{y} \pm 0.5 & c_{z} \pm 1 \\
d_{x} \pm 1.5 & d_{y} \pm 0.5 & d_{z} \pm 1.5
\end{array}\right]
$$

The criteria for defining the matrix in (3) were to ensure that the resulting trajectories were generally confined in layer 2, as they anatomically would be, and they varied approximately to an extent reported in the literature. A total of ten variations (V1-V10) of the nerve were defined by cubic spline interpolation of the four points in each case in Matlab. A minimum 


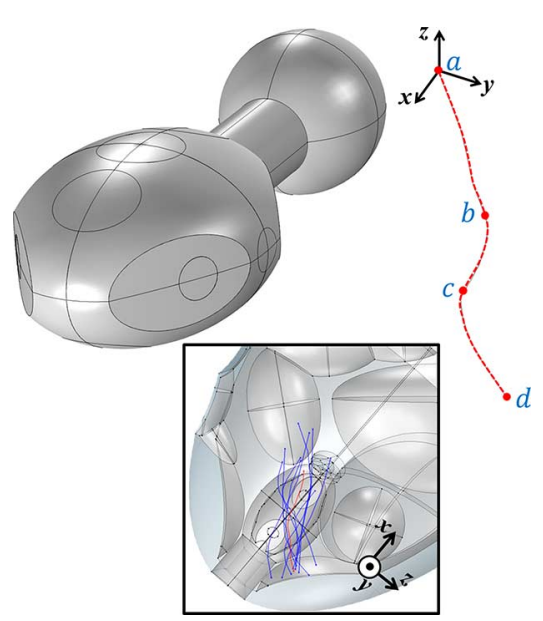

Fig. 6. Depicting the four points selected along the trajectory of the nerve found in the MRI study which were varied systematically to generate ten probable and possible variations of the trajectory. The inset shows the ten variations in dark blue and the main trajectory of the pudendal nerve in red in the FEM

length of $4 \mathrm{~cm}$ was enforced for the trajectories. Also, a minimum distance of $5 \mathrm{~mm}$ was enforced between all the similar points across different trajectories (e.g., between $a$ points). If the trajectory was placed outside layer 2 , the corresponding points were shifted or truncated manually after it was generated to place the whole length of the trajectory inside.

Anatomic constraints imposed on generating the variations were as follows:

$$
\begin{aligned}
& a_{x}<b_{x}<c_{x}<d_{x} \\
& a_{y}<b_{y}<c_{y}<d_{y} \\
& a_{z}<b_{z}<c_{z}<d_{z} .
\end{aligned}
$$

This would ensure that the overall trajectory consistently originates near the ischial spine and terminates near the organs it innervates. The inset of Fig. 6 shows the resulting variations. The dynamic response of each variation in terms of PA versus required current levels for $200 \mu \mathrm{s}$ stimulus pulses for 100 axons randomly placed along each of the trajectories was recorded as before for every configuration.

\section{G. Tripolar Configuration}

It was observed that the positions of depolarization may coincide along the trajectory of the nerve for the main trajectory and using all the bipolar configurations based on the AF study. From the bipolar configurations requiring the lowest levels of current, a tripolar configuration was formed for each variation and main trajectory of the nerve. PA versus required current was recorded as before.

\section{RESULTS}

\section{A. Approximations}

Fig. 7 compares the PA versus the required current levels for the main trajectory, the linear approximation of the nerve connecting the first and last points found in the MRI without EP, $\mathrm{PE}$, and EN tissue layers (Ln), the linear approximation of the nerve with all the tissue layers and four other fascicles around

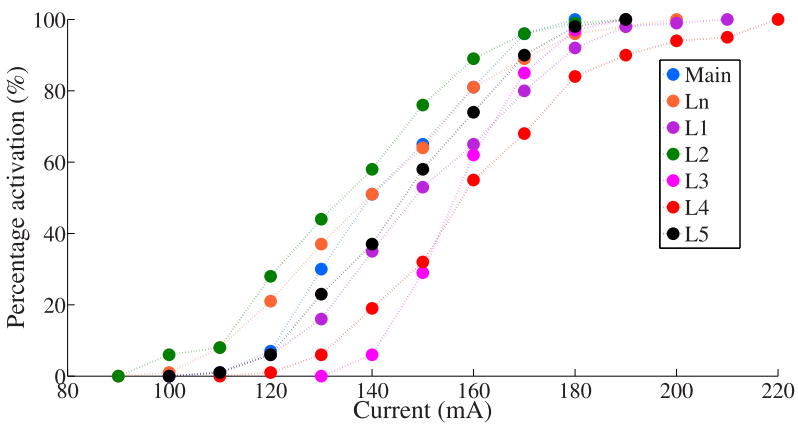

Fig. 7. Comparing the PA versus required current amplitude for the main trajectory of the nerve derived in the MRI study: linear approximation of this trajectory $(\mathrm{Ln})$, linear approximation of the trajectory with EP, PE, and EN tissue layers in place while four more fascicles are around the main fascicle. Also, the PA versus current amplitude for the fibers in the other four fascicles is shown.

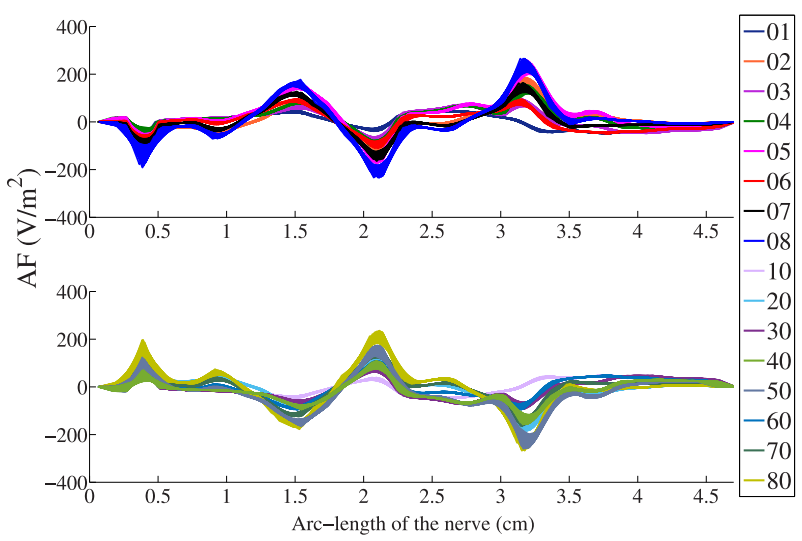

Fig. 8. AF for all the bipolar configurations and for an ensemble of 100 randomly distributed nerve fibers positioned randomly along the nerve trajectory obtained by the MRI study. The top plot shows all the forward configurations and the bottom plot all the reversed configurations. $1 \mathrm{~mA}$ current was applied.

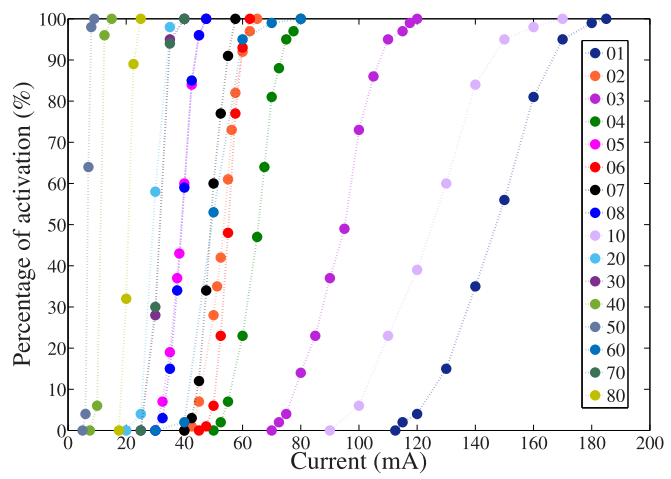

Fig. 9. PA versus current amplitude for all 16 bipolar electrode configurations for the main nerve trajectory using a $200-\mu$ s stimulus pulse. Electrode configurations 40 and 50 require the lowest stimulus current level.

it in place (L1), and those of the fibers in the other four fascicles (L2-L5). These are due to the original design of the stimulator and electrode configurations as used in [10] and shown in Fig. 1. While the PA versus the required current level for Ln closely follows that of the main trajectory, only about $5 \%$ variation is observed comparing those of $\mathrm{Ln}$ and $\mathrm{L} 1$ if $50 \%$ activation is considered. Overall, the variations in the required current do not exceed $15 \%$ for this level of activation. This variation should be considered in the subsequent comparison of different configurations which is insignificant as shown later in this paper. 

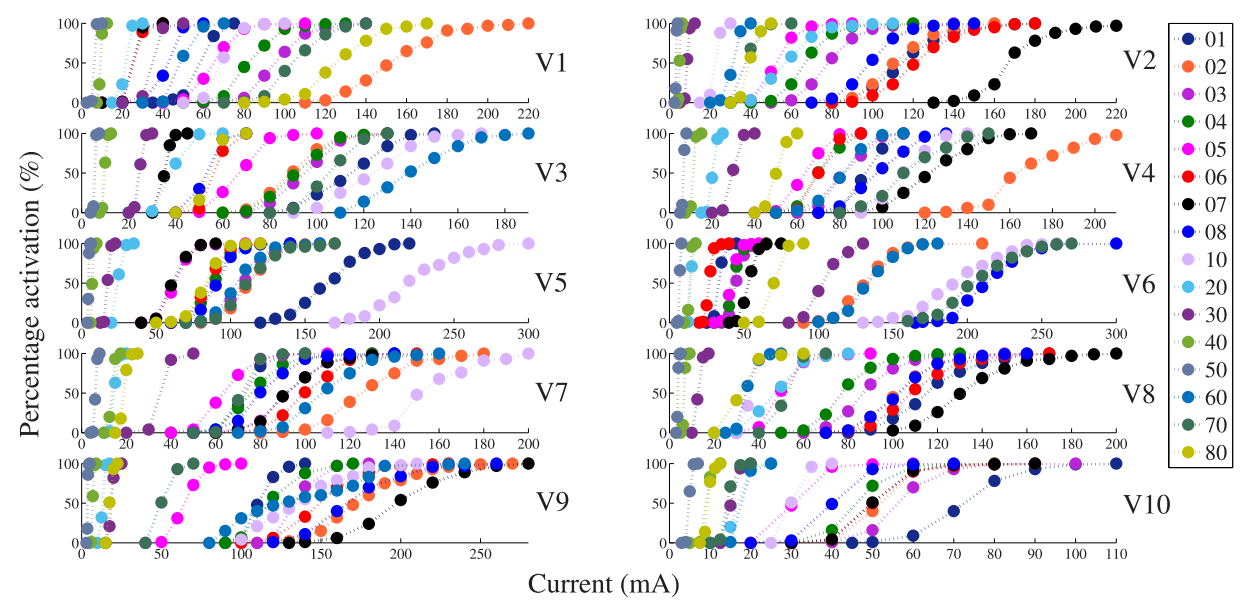

Fig. 10. PA versus the amplitude of the $200-\mu$ s stimulus pulse required for all the variations generated and shown in Fig. 6 . Distinctly, in all the variations electrode configurations 40 and 50 require the lowest current amplitude to activate the population of fibers.

\section{B. Optimization}

Based on the AF plots in Fig. 8, the depolarization positions along the course of the nerve coincide for all the forward configurations together, and reversed configurations together. Understandably, the AF plots of the reversed configurations are merely mirrors of the corresponding forward configurations with respect to the arc-length axis. Fig. 9 shows a large variation in the required current levels and that distinctly two electrode configurations (40 and 50) activate the nerve at much lower current levels. It is noted that there is not a direct correspondence between the peaks of the AF plots and the required current level. This is consistent with the findings of other investigations [30]. Hence, the AF concept should only be used as a tool to approximate the positions of depolarization and hyperpolarization along the nerve.

Running the simulation for ten different ensembles of 100 axons for the configuration requiring the lowest level of current (50), the limits of the PA versus current curve showed negligible variations $( \pm 1 \%)$ although the slope showed slight variations in some cases (a maximum of 9\%). This shows the validity of the 100 axon population as being sufficiently large to decide on the level of the current required.

Using electrode configuration 50 and for a 100 -axon random population, the $200-\mu$ s pulses were repeated at 5 and $35 \mathrm{~Hz}$. The amplitude was set to a level resulting in $100 \%$ activation for the first pulse. For $5 \mathrm{~Hz}$ pulse repetition frequency, the time between every subsequent pulse was sufficiently large to yield $100 \%$ activation in every pulse. However, for $35 \mathrm{~Hz}$ pulse repetition frequency, the PA was at $86 \% \pm 7 \%$ (mean \pm standard deviation) for 10 consecutive pulses.

\section{Nerve Variations}

As shown in Fig. 10 for all the variations of the nerve the same two configurations require considerably lower current levels (40 and 50). A striking result is that no other configuration demonstrates a low required current level consistently across all variations. The range of the required current also changes across variations and across the configurations although this variation is negligible for the two configurations (i.e., 40 and 50).

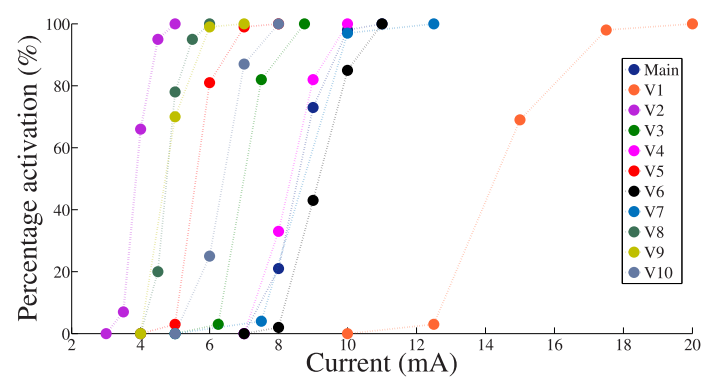

Fig. 11. PA versus required current for the (4-5) 0 configuration for all the variations using a $200-\mu$ s stimulus pulse. Relatively low current level is required for all the configurations. Only for V1 is there a slight increase in the required current.

\section{Tripolar Configurations}

The use of a tripolar configuration reduces the current spread [31]. In this case, the maximum voltage compliance for the stimulator would be reduced by providing two current paths.

Simulations showed that the position of depolarization coincides along the nerve for configurations in which electrode 0 was used as cathode and for those in which electrode 0 was used as anode. It was also verified that this was the case for all the variations and for the configurations under study (not shown here). Constituting a multipolar configuration out of the bipolar configurations, the resulting $\mathrm{AF}$ will be a linear addition of those of the constituent bipolar configurations. Thus, a relatively similar response may be expected of a tripolar configuration constituted of a linear addition of two bipolar configurations which show relatively similar PA versus current responses.

As shown in Fig. 11 the resulting tripolar configuration, (4-5)0 made from a linear addition of 40 and 50, consistently results in a low required current. Only in V1 does the current level slightly increase. Thus, without the need for an extensive trial of all possible configurations, a tripolar configuration in which the required current is relatively low was found.

\section{DISCUSSION}

In the hybrid models, the extracellular potential is simulated in a volume conductor model and is used in computational models of neurons and their segments to model the dynamic 
response of nerves to extracellular potential. These models have been used in a variety of applications. Namely, contributions by McIntyre [31], Danner [32], Howell [33], Capogrosso [13], Buston [34], Ladenbauer [35], and co-workers use such models to investigate different issues surrounding the stimulation of central neurons. Furthermore, studies by Kent [8], Raspopovic [15], Schiefer [26], Woock [9] and co-workers used hybrid models to study peripheral nerve stimulation.

In the study presented in this paper, trans-rectal stimulation of the pudendal nerve was investigated using similar methods. An approximate trajectory of the nerve with respect to a stimulator previously used by Craggs et al. [10] in situ was found using an MRI study. A volume conductor model of the stimulator in situ was made which was coupled to a model of the mammalian axon to investigate the dynamic response of the pudendal nerve to the trans-rectal stimulator. Plots of PA versus the amplitude of 200- $\mu$ s stimulus current pulses were used to characterize the dynamic response of the nerve to extracellular stimulation. It was shown that the variations in the results due to approximations made in the volume conductor are negligible compared to variations induced by implementing different electrode configurations. In addition, it was observed that the range of the current levels as reported based on the simulations was about $30 \%$ higher than those reported by Craggs et al. [10] $(n=6)$ which may be due to different reasons as explained here. Firstly, the distribution of fiber diameters may include larger fibers. It was also shown that the double layer cable model demonstrates slightly higher thresholds [36]. Moreover, at high levels of stimulus current as reported in [10], other excitable tissue near the stimulator may be activated, resulting in unknown reflexes with similar responses. Also, the number of subjects studied in [10] was not large enough. Therefore, any reported current value for a given electrode configuration should be considered relative to those of the other configurations.

Modifying the physical design of the stimulator slightly, a number of electrodes was incorporated on its surface. Two electrode configurations (40 and 50), with the cathodes facing caudally were identified to activate the nerve at much lower current levels. For low and high frequencies of stimulation it was shown that a sufficiently large population of axons may be activated. This indicates the possibility of using the stimulator to investigate clinically if neuromodulation at high frequencies could promote voiding using trans-rectal stimulation.

The study was repeated by considering ten anatomically possible and probable variations of the course of the nerve. In all cases activation of the fibers was achieved at much lower current levels using the same configurations when compared with the other electrode configurations. It was further demonstrated that a tripolar configuration can be formed using the two identified optimal bipolar configurations, which can activate fibers at equally low current levels. Given that the mentioned cathodes are next to each other, they can conveniently be merged to minimize the number of electrodes if equal current weighting is assigned to them. Thus, a bipolar configuration with a large cathode can be formed.

Having the cathode facing away from the nerve, while the anode facing it is at a closer distance, may be counterintuitive as it is generally expected to have activation near the cathode. However, in the case of the stimulator presented here, cathode and anode are at a distance away from the nerve $(\sim 2 \mathrm{~cm})$ and activation is the result of the overall generated field.

\section{CONCLUSION}

After SCI, various LUT dysfunctions may develop that can be fatal if left untreated. The treatment techniques range from drugs to intrusive surgeries. Pudendal nerve stimulation has been shown to offer the possibility of controlling bladder function after SCI. A wearable stimulator with surface electrodes and minimal side effects has the potential to greatly improve the quality of life for patients. Trans-rectal stimulation of the pudendal nerve may provide the opportunity for designing such a solution as the nerve passes in the vicinity of ano-rectal junction.

It has been shown in this paper, using computational models of a trans-rectal stimulator in situ and mammalian axon, that by optimizing the configuration of the electrodes the required current can be reduced significantly compared to the excessive levels previously reported in the literature [10]. This result indicates the possibility of designing a viable solution using trans-rectal stimulation of the pudendal nerve for controlling bladder function after SCI.

\section{APPENDIX A \\ BOUNDARY CONDITIONS}

In the $A C / D C$ module of COMSOL, Electric Currents physics in Stationary setting was selected. The software solves the following Maxwell's equations to simulate the electric potential in the model:

$$
\begin{aligned}
& \nabla \cdot J=Q_{j} \\
& J=\sigma E+J_{e} \\
& E=-\nabla V
\end{aligned}
$$

where $J$ is the current density, $Q_{j}$ is current source, $E$ is the electric field, and $J_{e}$ is the external current density. By setting $Q_{j}$ and $J_{e}$ to zero everywhere in the model, quasi-static approximation of Maxwell's equations was solved.

\section{APPENDIX B \\ CONDUCTIVITY TENSOR}

Assuming a local Cartesian coordinate system for the nerve $\left(x^{\prime} y^{\prime} z^{\prime}\right)$ in which the nerve trajectory is elongated along the $x^{\prime}$ axis, the conductivity of EN is defined by the tensor shown as follows:

$$
\sigma^{\prime}=\left[\begin{array}{ccc}
\sigma_{L} & 0 & 0 \\
0 & \sigma_{T} & 0 \\
0 & 0 & \sigma_{T}
\end{array}\right] .
$$

The variables $x^{\prime}, y^{\prime}$ and $z^{\prime}$ can be found based on $x, y$, and $z$ in the global system as follows:

$$
\begin{aligned}
& x^{\prime}=\left[\begin{array}{ccc}
\cos \theta_{2} & 0 & \sin \theta_{2} \\
0 & 1 & 0 \\
-\sin \theta_{2} & 0 & \cos \theta_{2}
\end{array}\right]\left[\begin{array}{ccc}
\cos \theta_{1} & -\sin \theta_{2} & 0 \\
\sin \theta_{1} & \cos \theta_{1} & 0 \\
0 & 0 & 1
\end{array}\right]\left[\begin{array}{l}
1 \\
0 \\
0
\end{array}\right] \\
& y^{\prime}=\left[\begin{array}{ccc}
\cos \theta_{1} & -\sin \theta_{1} & 0 \\
\sin \theta_{1} & \cos \theta_{1} & 0 \\
0 & 0 & 1
\end{array}\right]\left[\begin{array}{l}
0 \\
1 \\
0
\end{array}\right] \\
& z^{\prime}=x^{\prime} \times y^{\prime}
\end{aligned}
$$


where $\theta_{1}$ and $\theta_{2}$ are the angles of rotation around $z$ and $y^{\prime}$ axes, respectively. Then, the transformation matrix $Q$ can be formed

$$
Q=\left[\begin{array}{ccc}
x_{x}^{\prime} & x_{y}^{\prime} & x_{z}^{\prime} \\
y_{x}^{\prime} & y_{y}^{\prime} & y_{z}^{\prime} \\
z_{x}^{\prime} & z_{y}^{\prime} & z_{z}^{\prime}
\end{array}\right]
$$

Subsequently, the conductivity tensor in global coordinates can be found as follows:

$$
\sigma=Q \sigma^{\prime} Q^{T}
$$

\section{ACKNOWLEDGMENT}

The authors would like to thank Dr. Kirkham for his help in performing the MRI and Prof. Donaldson, Dr. Vanhostenberge, and Dr. Mosse for their valuable involvement in the project. The authors would like to also thank Dr. Carnevale for his suggestions on the Neuron model.

\section{REFERENCES}

[1] C. J. Fowler, D. Griffiths, and W. C. de Groat, "The neural control of micturition," Nat. Rev. Neurosci., vol. 9, no. 6, pp. 453-466, 2008.

[2] C. Sacomani, F. Trigo-Rocha, C. Gomes, J. Greve, T. Barros, and S. Arap, "Effect of the trauma mechanism on the bladder-sphincteric behavior after spinal cord injury," Spinal Cord, vol. 41, no. 1, pp. 12-15, 2003.

[3] R. A. Gaunt and A. Prochazka, "Control of urinary bladder function with devices: Successes and failures," Prog. Brain Res., vol. 152, pp. 163-194, 2006.

[4] P. Sanders, M. Ijzerman, M. Roach, and K. Gustafson, "Patient preferences for next generation neural prostheses to restore bladder function," Spinal Cord, vol. 49, no. 1, pp. 113-119, 2011.

[5] G. Brindley, "The first 500 patients with sacral anterior root stimulator implants: General description," Spinal Cord, vol. 32, no. 12, pp. 795-805, 1994.

[6] W. C. de Groat, D. Griffiths, and N. Yoshimura, "Neural control of the lower urinary tract," Compr. Physiol., vol. 5, no. 1, pp. 327-396, 2015.

[7] C. Tai, J. Wang, X. Wang, W. C. de Groat, and J. R. Roppolo, "Bladder inhibition or voiding induced by pudendal nerve stimulation in chronic spinal cord injured cats," Neurorol. Urodyn., vol. 26, no. 4, pp. $570-577,2007$.

[8] A. R. Kent and W. M. Grill, "Model-based analysis and design of nerve cuff electrodes for restoring bladder function by selective stimulation of the pudendal nerve," J. Neural Eng., vol. 10, no. 3, p. 036010, 2013.

[9] J. P. Woock, P. B. Yoo, and W. M. Grill, "Finite element modeling and in vivo analysis of electrode configurations for selective stimulation of pudendal afferent fibers," BMC Urol., vol. 10, no. 1, p. 11, 2010.

[10] M. Craggs, N. Edirisinghe, B. Leaker, J. Susser, M. Al-Mukhtar, and N. Donaldson, "Conditional neuromodulation using trans-rectal stimulation in spinal cord injury," Neurourol. Urodyn., vol. 28, no. 7, pp. $836-837,2009$

[11] M. Craggs, "Neuromodulation device for pelvic dysfunction," U.S 8,644,938, Feb. 4, 2014.

[12] A. Shafik, M. El-Sherif, A. Youssef, and E. S. Olfat, "Surgical anatomy of the pudendal nerve and its clinical implications," Clin. Anat., vol. 8 , no. 2, pp. 110-115, 1995.

[13] M. Capogrosso, N. Wenger, S. Raspopovic, P. Musienko, J. Beauparlant, L. B. Luciani, G. Courtine, and S. Micera, "A computational model for epidural electrical stimulation of spinal sensorimotor circuits," J. Neurosci., vol. 33, no. 49, pp. 19326-19340, 2013.

[14] A. N. Shiraz, A. Demosthenous, and A. Vanhoestenberghe, "Towards an optimized wearable neuromodulation device for urinary incontinence," in Proc. 19th IEEE Int. Conf. Electronics, Circuits Syst. (ICECS), Seville, Spain, 2012, pp. 25-28.

[15] S. Raspopovic, M. Capogrosso, and S. Micera, "A computational model for the stimulation of rat sciatic nerve using a transverse intrafascicular multichannel electrode," IEEE Trans. Neural Syst. Rehabil. Eng., vol. 19, no. 4, pp. 333-344, Jul. 2011.

[16] C. Gabriel, S. Gabriel, and E. Corthout, "The dielectric properties of biological tissues: I. Literature survey," Phys. Med. Biol., vol. 41, no. 11, pp. 2231-2249, 1996

[17] S. Gabriel, R. Lau, and C. Gabriel, "The dielectric properties of biological tissues: II. Measurements in the frequency range $10 \mathrm{~Hz}$ to 20 GHz," Physics Medicine Biol., vol. 41, no. 11, p. 2251, 1996.
[18] S. Gabriel, R. Lau, and C. Gabriel, "The dielectric properties of biological tissues: III. Parametric models for the dielectric spectrum of tissues," Phys. Med. Biol., vol. 41, no. 11, pp. 2251-2269, 1996.

[19] P. Suhel, P. Vrtacnik, and M. Trlep, "Bioimpedance measurement in the lower urinary tract: Numerical calculation of the potential and current distribution," in Proc. IEEE 17th Annu. Conf. Eng. Medicine Biol. Soc. (EMBC), Montreal, Canada, 1995, vol. 2, pp. 1523-1524.

[20] C. M. Davies, C. M. Ferguson, C. Kaucner, M. Krogh, N. Altavilla, D. A. Deere, and N. J. Ashbolt, "Dispersion and transport of Cryptosporidium oocysts from fecal pats under simulated rainfall events," Appl. Environ. Microbiol., vol. 70, no. 2, pp. 1151-1159, 2004.

[21] C. C. McIntyre, A. G. Richardson, and W. M. Grill, "Modeling the excitability of mammalian nerve fibers: Influence of afterpotentials on the recovery cycle," J. Neurophysiol., vol. 87, no. 2, pp. 995-1006, 2002.

[22] G. Schalow, G. Zäch, and R. Warzok, "Classification of human peripheral nerve fibre groups by conduction velocity and nerve fibre diameter is preserved following spinal cord lesion,"J. Auton. Nerv. Syst., vol. 52, no. 2, pp. 125-150, 1995 .

[23] M. L. Hines and N. T. Carnevale, "The NEURON simulation environment," Neural Comput., vol. 9, no. 6, pp. 1179-1209, 1997.

[24] M. L. Hines, T. Morse, M. Migliore, N. T. Carnevale, and G. M. Shepherd, "ModelDB: A database to support computational neuroscience," J. Comput. Neurosci., vol. 17, no. 1, pp. 7-11, 2004.

[25] J. Erlanger and E. A. Blair, "Comparative observations on motor and sensory fibers with special reference to repetitiousness," Amer. J. Physiol_Legacy Content, vol. 121, no. 2, pp. 431-453, 1938.

[26] M. A. Schiefer, R. J. Triolo, and D. J. Tyler, "A model of selective activation of the femoral nerve with a flat interface nerve electrode for a lower extremity neuroprosthesis," IEEE Trans. Neural Syst. Rehabil. Eng., vol. 16, no. 2, pp. 195-204, Mar. 2008.

[27] F. Rattay, "Analysis of models for extracellular fiber stimulation," IEEE Trans. Biomed. Eng., vol. 36, no. 7, pp. 676-682, Jul. 1989.

[28] P. Kovacs, H. Gruber, J. Piegger, and G. Bodner, "New, simple, ultrasound-guided infiltration of the pudendal nerve," Dis. Colon Rectum, vol. 44, no. 9, pp. 1381-1385, 2001.

[29] M. Schenck, C. Schenck, H. Rübben, M. Stuschke, T. Schneider, A. Eisenhardt, and R. Rossi, "Pudendal nerve block in HDRbrachytherapy patients: Do we really need general or regional anesthesia?," World J. Urol., vol. 31, no. 2, pp. 417-421, 2013.

[30] C. M. Zierhofer, "Analysis of a linear model for electrical stimulation of axons - Critical remarks on the "activating function concept"," IEEE Trans. Biomed. Eng., vol. 48, no. 2, pp. 173-184, Feb. 2001

[31] C. C. McIntyre and W. M. Grill, "Extracellular stimulation of central neurons: Influence of stimulus waveform and frequency on neuronal output," J. Neurophysiol., vol. 88, no. 4, pp. 1592-1604, 2002.

[32] S. M. Danner, U. S. Hofstoetter, J. Ladenbauer, F. Rattay, and K. Minassian, "Can the human lumbar posterior columns be stimulated by transcutaneous spinal cord stimulation? A modeling study," Artif. Organs, vol. 35, no. 3, pp. 257-262, 2011.

[33] B. Howell, S. P. Lad, and W. M. Grill, "Evaluation of intradural stimulation efficiency and selectivity in a computational model of spinal cord stimulation," PloS One, vol. 9, no. 12, p. e114938, 2014.

[34] C. R. Butson and C. C. McIntyre, "Tissue and electrode capacitance reduce neural activation volumes during deep brain stimulation," Clin. Neurophysiol., vol. 116, no. 10, pp. 2490-2500, 2005.

[35] J. Ladenbauer, K. Minassian, U. S. Hofstoetter, M. R. Dimitrijevic, and F. Rattay, "Stimulation of the human lumbar spinal cord with implanted and surface electrodes: A computer simulation study," IEEE Trans. Neural Syst. Rehabil. Eng., vol. 18, no. 6, pp. 637-645, Nov. 2010.

[36] A. Richardson, C. McIntyre, and W. Grill, "Modelling the effects of electric fields on nerve fibres: Influence of the myelin sheath," Med. Biol. Eng. Comput., vol. 38, no. 4, pp. 438-446, 2000.

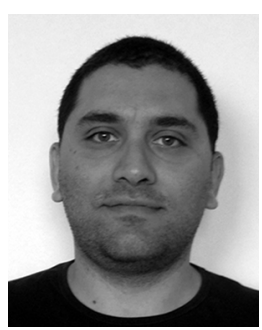

Arsam N. Shiraz (S'13) was born in Tehran, Iran in 1983. He received a first-class M.Eng. degree in electronic and electrical engineering from University College London (UCL), London, U.K., in 2011. He is currently working towards the Ph.D. degree in the Analog and Biomedical Electronics Group, UCL.

His research interests are the design and development of medical devices (sensing and stimulation) and bio-modelling (FEM, neural models and bio-potential simulation).

Mr. Shiraz was on the Dean's list and was awarded the Departmental Outstanding Student Prize. He was awarded a UCL Overseas Research Scholarship and a UCL Faculty of Engineering Postgraduate Research Scholarship, in 2011. 


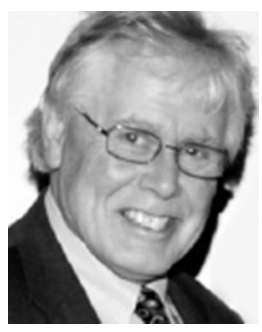

Michael Craggs became Emeritus Professor of applied clinical neurophysiology in the Division of Surgery and Interventional Sciences, University College London (UCL), London, U.K., in 2009. His research profile is extensive, stretching back over 40 years, including a long employment in the Medical Research Council (MRC) as a Senior Scientist with Professor Giles Brindley at the Neurological Prostheses Unit, as Senior Lecturer at the Institute of Psychiatry, and as Principal Clinical Scientist at UCL Hospitals. For the last 15 years before retiring he was the Director of Neurospinal Research and Consultant Clinical Scientist for the London Spinal Cord Injury Centre, Royal National Orthopaedic Hospital NHS Trust, Stanmore, U.K.

Brian Leaker is a Consultant Nephrologist with an interest in nephro-urology and treatment of complex urinary disorders. He is an accredited clinical investigator for Phase I and high-risk clinical studies (MHRA). He has established a specialist clinical trials company, Nephro-Urology Clinical Trials (NUCT) Ltd., which conducts clinical trials of novel drugs and devices to treat incontinence and disorders of the urinary tract. NUCT Ltd. holds a worldwide patent for the wearable pudendal stimulation device.

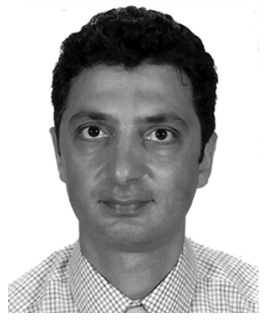

Andreas Demosthenous (S'94-M'99-SM'05) received the B.Eng. degree in electrical and electronic engineering from the University of Leicester, Leicester, U.K., in 1992, the M.Sc. degree in telecommunications technology from Aston University, Birmingham, U.K., in 1994, and the Ph.D. degree in electronic and electrical engineering from University College London (UCL), London, U.K., in 1998.

$\mathrm{He}$ is currently a Professor in the Department of Electronic and Electrical Engineering, UCL, where he leads the Analog and Biomedical Electronics Group. He has published more than 190 articles in journals and international conference proceedings. His current research interests include analog and mixed-signal integrated circuits for biomedical, sensor, and signal-processing applications.

Dr. Demosthenous is a Fellow of the Institution of Engineering and Technology and a Chartered Engineer. He is the Deputy Editor-in-Chief of the IEEE TRANSACTIONS ON CIRCUITS AND SYSTEMS II: EXPRESS BRIEFS (term 2014 to 2015) and an Associate Editor of the IEEE TRANSACTIONS ON BIOMEDICAL CirCUITS AND SYSTEMS. He has recently been appointed the Editor-in-Chief of the IEEE TRANSACtions on Circuits and Systems II: Regular PAPERS (term 2016 to 2017). He is on the International Advisory Board of Physiological Measurement, Institute of Physics. He is a Member of the Technical Programme Committee of several IEEE conferences including European SolidState Circuits (ESSCIRC). He was on the Organizing Committee of the 2013 IEEE Biomedical Circuits and Systems Conference (BioCAS). 